\title{
Serum Bicarbonate Is Associated with Heart Failure in the Multi-Ethnic Study of Atherosclerosis
}

\author{
Jessica B. Kendrick ${ }^{a, b}$ Leila Zelnick ${ }^{c}$ Michel B. Chonchol ${ }^{a}$ David Siscovick $^{\mathrm{e}}$ \\ Andrew N. Hoofnagled Joachim H. Ix ${ }^{f}$ Mark Sarnak $^{g}$ Michael G. Shlipak ${ }^{\mathrm{h}}$ \\ Bryan Kestenbaum ${ }^{c}$ Ian H. de Boer ${ }^{c}$ \\ aDivision of Renal Diseases and Hypertension, University of Colorado School of Medicine, Aurora, CO, \\ ${ }^{b}$ Denver Health Medical Center, Denver, CO, 'Division of Nephrology, Kidney Research Institute, and \\ ${ }^{\mathrm{d}}$ Department of Laboratory Medicine, University of Washington, Seattle, WA, eNew York Academy of Medicine, \\ New York, NY, fDivision of Nephrology and Hypertension, Department of Medicine, University of California, \\ San Diego, CA, ${ }^{9}$ Division of Nephrology, Tufts Medical Center, Boston, MA, and hivision of General Internal \\ Medicine, San Francisco Veterans Affairs Medical Center and University of California, San Francisco, CA, USA
}

\section{Key Words}

Cardiovascular disease $\cdot$ Bicarbonate $\cdot$ Heart failure .

Arterial stiffness $\cdot$ Left ventricular mass

\begin{abstract}
Background: Low serum bicarbonate concentrations are associated with mortality and kidney disease progression. Data regarding associations between bicarbonate and cardiovascular disease (CVD) are scarce. Methods: We performed a cohort study of 6,229 adult participants from the Multi-Ethnic Study of Atherosclerosis, a community-based cohort free of CVD at baseline. Serum bicarbonate was measured at baseline. Cardiovascular outcomes were defined as: (1) subclinical CVD (left ventricular mass [LVM] and aortic pulse pressure [PP] measured at baseline), (2) incident atherosclerotic cardiovascular events (CVE; composite of myocardial infarction, resuscitated cardiac arrest, stroke, coronary heart disease death, and stroke death), and (3) incident
\end{abstract}

heart failure. Results: During a median (interquartile range) follow-up of 8.5 (7.7-8.6) years, 331 (5.3\%) participants had an incident CVE and 174 (2.8\%) developed incident heart failure. We stratified analyses by use of diuretics because we observed a significant interaction between diuretic use and bicarbonate with study outcomes. Among diuretic nonusers, with adjustment, bicarbonate $\geq 25 \mathrm{mEq} / \mathrm{L}$ was associated with an estimated $3.0 \mathrm{~g}$ greater LVM $(95 \% \mathrm{Cl} 0.5-5.0)$ and $1.0 \mathrm{~mm} \mathrm{Hg}$ higher aortic PP (95\% Cl 0.4-2.0) compared to bicarbonate 23-24 mEq/L. Each $1 \mathrm{mEq} / \mathrm{L}$ of bicarbonate increase was associated with a $13 \%$ higher risk of incident heart failure (hazards ratio 1.13, 95\% Cl 1.01-2.11). Among diuretic users, higher bicarbonate was not associated with CVD. Bicarbonate was not associated with incident atherosclerotic CVE irrespective of diuretic use. Conclusion: Among nonusers of diuretics in a large community-based study, higher serum bicarbonate concentrations are associated with subclinical CVD and new heart failure.

(c) 2016 S. Karger AG, Basel

\section{KARGER}

E-Mail karger@karger.com

www.karger.com/ajn (c) 2016 S. Karger AG, Basel

Assoc. Prof. Jessica B. Kendrick, MD, MPH

Division of Renal Diseases and Hypertension, University of Colorado Denver

Denver Health Medical Center 660 Bannock Street, Mail Code 4000

Denver, CO 80204 (USA)

E-Mail Jessica.Kendrick@ucdenver.edu 


\section{Introduction}

The leading cause of death in both men and women in the United States is cardiovascular disease (CVD). While interventions aimed at traditional cardiovascular risk factors have resulted in improved outcomes [1], there is considerable interest in identifying novel cardiovascular risk factors. Identification of new risk factors will improve our understanding of the disease and may account for cases of CVD that cannot be explained by traditional risk factors. Metabolic acidosis, as reflected by a low serum bicarbonate concentration, may be a modifiable risk factor for CVD in patients with and without kidney disease.

Metabolic acidosis results in numerous deleterious effects such as chronic inflammation and impaired myocardial function [2]. Recent studies have also found that metabolic acidosis results in activation of the renin-angiotensin aldosterone system and vascular endothelial dysfunction [3]. Epidemiologic studies have found that lower serum bicarbonate concentrations are associated with incident and prevalent hypertension [4-6], insulin resistance $[7,8]$, kidney disease progression [9-16], and mortality [14-18]. Collectively these data suggest that metabolic acidosis may be a risk factor for CVD. However, 2 studies have reported the opposite [12, 19]. Both studies found that higher serum bicarbonate concentrations were associated with increased risk of heart failure in patients with chronic kidney disease (CKD) [12, 19]. Effects of alkalosis on vascular calcification and regulatory proteins in the heart were postulated as potential mechanisms underlying these associations. It is unknown whether bicarbonate concentrations are associated with CVD in patients without $\mathrm{CKD}$, for whom the causes and consequences of altered bicarbonate concentration may differ.

We examined associations between serum bicarbonate concentration and clinical cardiovascular events (CVE) and subclinical CVD in a prospective, multiethnic cohort of 6,229 individuals who were initially free of clinical CVD. A priori, we hypothesized that lower serum bicarbonate levels would be associated with a higher risk of CVD.

\section{Methods}

\section{Study Population}

The Multi-Ethnic Study of Atherosclerosis (MESA) is a prospective cohort study of CVD among 6,814 community living adults. Details of the design and recruitment for MESA have been published previously [20]. Briefly, MESA recruited adults aged 4584 years from July 2000 through July 2002. Participants were re- cruited from Baltimore, MD, Chicago IL, Forsyth County, NC, Los Angeles County, CA, Northern Manhattan and the Bronx, NY, and St. Paul MN. MESA excluded participants who had any previous diagnosis of CVD, defined by self-reported diagnosis of myocardial infarction, angina, nitroglycerin use, atrial fibrillation, transient ischemic attack, stroke, heart failure, angioplasty, coronary artery bypass grafting, valve replacement, pacemaker or defibrillator, or any other heart or arterial surgery. Institutional review board approval was obtained for each site and all participants gave informed consent. For this study, we excluded participants who were missing one or more of the following: baseline measurements of serum bicarbonate $(n=381)$, creatinine $(n=25)$, cystatin $\mathrm{C}(n=58)$, urine albumin to creatinine ratio $(n=39)$, data on CVE $(n=5)$, education $(n=23)$, smoking status $(n=22)$, self-reported emphysema $(n=1)$, systolic blood pressure $(n=3)$, C-reactive protein $(n=52)$, triglycerides $(n=23)$, low-density lipoprotein cholesterol $(n=113)$, high density lipoprotein cholesterol $(n=26)$, or medication information $(n=3)$. This resulted in a final analytic sample of 6,229 participants (91.4\%).

\section{Measurement of Serum Bicarbonate Concentrations}

MESA study personnel collected baseline serum samples in the morning after an overnight fast. Serum was frozen at $-80^{\circ} \mathrm{C}$, and bicarbonate was measured from thawed specimens using a $\mathrm{pH}$ rate-of-change method with an ion selective electrode on a Beckman DxC automated chemistry analyzer at the University of Washington. The interassay coefficient of variation across the study was $2.98-3.19 \%$ [16].

\section{Outcomes}

\section{Subclinical Disease}

The outcomes of interest were aortic pulse pressure (PP) and left ventricular (LV) mass. PulseWave CR-2000 Research CardioVascular Profiling Instrument was used to record 30-s radial artery waveform recordings [21]. The recordings were digitized at $200 \mathrm{~Hz}$ and exported for processing using software by Matlab (The Mathworks; Natick, MA, USA). Assuming no brachial-to-radial amplification, the radial pressure waveform was calibrated with brachial systolic and diastolic pressures. To obtain a central pressure waveform, a generalized transfer function was applied to the radial pressure waveform. Aortic PP was computed as resting aortic systolic blood pressure minus aortic diastolic blood pressure.

LVM was calculated from cardiac MRI images in a subset of participants $(n=4,603)$ [22]. MESA personnel performed cardiac MRIs using scanners with 1.5-T magnets. LVM was calculated using commercially available software (MASS 4.2; Leiden, The Netherlands) by central readers blinded to other study data. The intraclass correlation coefficient for LVM was $0.98(n=75)$ [22].

\section{Cardiovascular Events}

CVE were collected by study personnel through telephone interviews and scheduled follow-up examinations. Hospitalization records, outpatient visits, and/or death certificates were collected and reviewed by 2 independent study physicians blinded to study data. Incident heart failure was defined as probable if the diagnosed by a physician plus the patient had received medical treatment for heart failure. The MESA Events Committee defined definite heart failure as a diagnosis of heart failure by a physician plus treatment for heart failure as well as either echocardiographic or chest X-ray evidence of heart failure. Coronary heart disease was 
defined as definite angina, probable angina if followed by percutaneous coronary intervention or coronary artery bypass grafting, myocardial infarction, resuscitated cardiac arrest, or coronary artery disease death. Ischemic stroke was defined as a focal neurological deficit lasting $>24 \mathrm{~h}$ or stroke symptoms lasting $<24 \mathrm{~h}$ with clinically relevant lesion on brain imaging. As in previous MESA studies [23-25], we evaluated "hard atherosclerotic CVD," which in MESA was defined as myocardial infarction, resuscitated cardiac arrest, stroke, coronary heart disease death, and stroke death.

\section{Covariate Data}

Study personnel collected demographic and health information on study participants using standardized questionnaires. Medication usage was determined by questionnaire and review of pill bottles. Age, race/ethnicity, and gender were self-identified. Participant's height and weight were measured and body mass index (BMI) was calculated as weight $/$ height $^{2}\left(\mathrm{~kg} / \mathrm{m}^{2}\right)$. All measurements were completed with participants wearing light clothing and no shoes. At each examination, blood pressure was measured with a Dinamap Pro100 (Critikon, Tampa, FL, USA) in a seated position 3 times. The second and third measurements were averaged and recorded as the blood pressure for that examination. Smoking was categorized as never, former, or current smoker. Diabetes was defined as fasting blood glucose $\geq 126 \mathrm{mg} / \mathrm{dL}$ or use of glucose lowering medication. A history of emphysema was determined by self-report. Percent emphysema was evaluated at the first examination for some participants $(n=3,622)$. Percent emphysema was calculated by trained readers blinded to study treatment from cardiac CT scans performed at baseline following a standardized protocol. Percent emphysema was defined as the percentage of lung voxels below -950 Hounsfield units. Dietary protein density was calculated from a 120 -item food frequency questionnaire that was performed at the baseline examination [26].

Fasting morning blood samples were drawn at each clinic visit and shipped to the MESA central laboratory. Serum creatinine was measured by rate reflectance spectrophotometry using thin-film adaptation of the creatinine amidohydrolase method on the VITROS analyzer (Johnson and Johnson clinical Diagnostics Inc) and calibrated to the Cleveland Clinic. Cystatin $\mathrm{C}$ was measured by a particle-enhanced immunonephelometric assay ( $\mathrm{N}$ Latex Cystatin C, Siemens AG, Munich, Germany). The CKD Epidemiology Collaboration creatinine-cystatin C equation was used to estimate glomerular filtration rate (GFR). Urine albumin and creatinine were measured from spot morning collections using nephelometry and the rate Jaffe reaction, respectively. Lipid panels and C-reactive protein were measured using standard techniques at the central laboratory.

\section{Statistical Analysis}

Serum bicarbonate concentrations were examined as a continuous variable and in clinically significant categories: $<21,21-22$, $23-24$, and $\geq 25 \mathrm{mEq} / \mathrm{L}$. For this analysis, a bicarbonate concentration of $23-24 \mathrm{mEq} / \mathrm{L}$ was chosen as the reference group because it is within the normal clinical range and contained the mean and median values for our study population. Multivariable linear regression models and Cox proportional hazards models were used to examine associations of serum bicarbonate concentration with subclinical disease and CVE, respectively. Prior to analysis, we considered variables that may be associated with serum bicarbonate concentration and CVD as potential covariates. Our basic model included adjustment for age, race/ethnicity, gender, attained education level, and MESA site. Model 2 included the covariates in model 1 plus diabetes, self-reported emphysema, BMI, systolic blood pressure, smoking, antihypertensive medications, lipid-lowering medications, estimated GFR (eGFR), C-reactive protein, triglycerides, high-density lipoprotein cholesterol, lowdensity lipoprotein cholesterol, and urine albumin to creatinine ratio. Since protein intake is the major contributor to dietary acid load [27], we evaluated an additional model adjusting for dietary protein density. We evaluated for heterogeneity of bicarbonateCVD associations by diuretic use because diuretics have large effects on serum bicarbonate concentrations. Analyses were stratified by diuretic use since we observed a significant interaction between bicarbonate levels and diuretic use examining incident heart failure. Statistical analyses were conducted using SAS statistical software, and $p$ values $<0.05$ were considered statistically significant for all analyses including interaction terms.

\section{Results}

The mean (SD) age was $62(10.3)$ years, and mean serum bicarbonate concentration was 23.1 (1.8) $\mathrm{mEq} / \mathrm{L}$ (Table 1). The median (interquartile range, IQR) and range of serum creatinine was $0.9(0.8-1.1) \mathrm{mg} / \mathrm{dL}$ and $0.2-6.0 \mathrm{mg} / \mathrm{dL}$, respectively. The median (IQR) and range of eGFR was $78.2(67.2-89.1) \mathrm{mL} / \mathrm{min} / 1.73 \mathrm{~m}^{2}$ and $6.5-$ $175.9 \mathrm{~mL} / \mathrm{min} / 1.73 \mathrm{~m}^{2}$, respectively. Participants with bicarbonate $<21 \mathrm{mEq} / \mathrm{L}$ were more likely to be younger and to have diabetes, higher BMI, higher eGFR, and urine albumin to creatinine ratios. Participants with bicarbonate levels $\geq 25 \mathrm{mEq} / \mathrm{L}$ were more likely to use antihypertensive medications and diuretics. They were also more likely to be female gender and Caucasian, and attained higher levels of education.

\section{Associations with Subclinical CVD}

There was no significant interaction between diuretic use and bicarbonate with the outcome of LVM ( $p=0.9$ for both categorical and continuous models) and aortic PP ( $p>0.80$ for both categorical and continuous models). Higher serum bicarbonate concentrations were associated with greater LVM and higher aortic PP in participants not using diuretics (Table 2). In participants not using diuretics $(n=5,216)$, bicarbonate $\geq 25 \mathrm{mEq} / \mathrm{L}$ was associated with a $3.0 \mathrm{~g}$ higher LVM compared to $23-24 \mathrm{mEq} / \mathrm{L}$, adjusting for covariates in the fully adjusted model, and each $1 \mathrm{mEq} / \mathrm{L}$ bicarbonate increase was associated with a $0.7 \mathrm{~g}$ higher LVM. Similarly, in participants not on diuretics, bicarbonate $\geq 25 \mathrm{mEq} / \mathrm{L}$ was associated with higher aortic PP compared to a concentration of $23-24 \mathrm{mEq} / \mathrm{L}$ ( $\beta=1.0$ $\mathrm{mm} \mathrm{Hg}, 95 \% \mathrm{CI} 0.4-2.0$ ) in the fully adjusted model, and each $1 \mathrm{mEq} / \mathrm{L}$ bicarbonate increase in concentration was 
Table 1. Characteristics of study population by serum bicarbonate concentration



Values are expressed as mean (SD) unless otherwise specified.

BMI, body mass index; LDL, low density lipoprotein cholesterol; HDL, high-density lipoprotein cholesterol; eGFR, estimated glomerular filtration rate.

associated with a $0.3 \mathrm{~mm} \mathrm{Hg}$ higher aortic PP. There was no significant association between serum bicarbonate concentration and LVM or aortic PP among the 1,013 participants using diuretics. Results were unchanged after further adjustment for dietary protein density (model 3).

\section{Associations with Incident CVE and Heart Failure}

Three hundred thirty-one participants had an incident atherosclerotic CVD event during a median (IQR) follow-up of 8.5 (7.6-8.6) years. There were 174 incident heart failure events during a median (IQR) follow-up for 
Table 2. Associations of bicarbonate concentration with subclinical CVD



Model 1: adjusted for age, race/ethnicity, gender, education, and MESA site.

Model 2: adjusted for covariates in model 1 plus diabetes, self-reported emphysema, body mass index, systolic blood pressure, smoking, high-density lipoprotein cholesterol, low-density lipoprotein cholesterol, triglycerides, eGFR, C-reactive protein, urine albumin to creatinine ratio, use of antihypertensive medications, use of lipid-lowering medications.

Model 3: adjusted for covariates in model 2 plus dietary protein density.

* $p$ value is for continuous comparison.

** Additionally adjusted for mean arterial pressure.

8.5 (7.7-8.6) years. There was a significant interaction between serum bicarbonate and diuretic use when examining incident heart failure $(p=0.03$ in model 3 when bicarbonate was examined categorically and 0.003 in model 3 when bicarbonate was examined continuously). There was no significant association between serum bicarbonate and incident atherosclerotic CVD events among either users or nonusers of diuretics (Table 3). Among par- ticipants using diuretics, bicarbonate $>25 \mathrm{mEq} / \mathrm{L}$ was associated with lower risk of incident heart failure in the fully adjusted model compared with $23-24 \mathrm{mEq} / \mathrm{L}$, although the association was not significant in the continuous model. Among nonusers of diuretics, in the continuous model, each $1 \mathrm{mEq} / \mathrm{L}$ increase in serum bicarbonate was significantly associated with a $13 \%$ increased risk of incident heart failure in the fully adjusted model; findings 
Table 3. Associations of bicarbonate concentration with incident CVE and heart failure

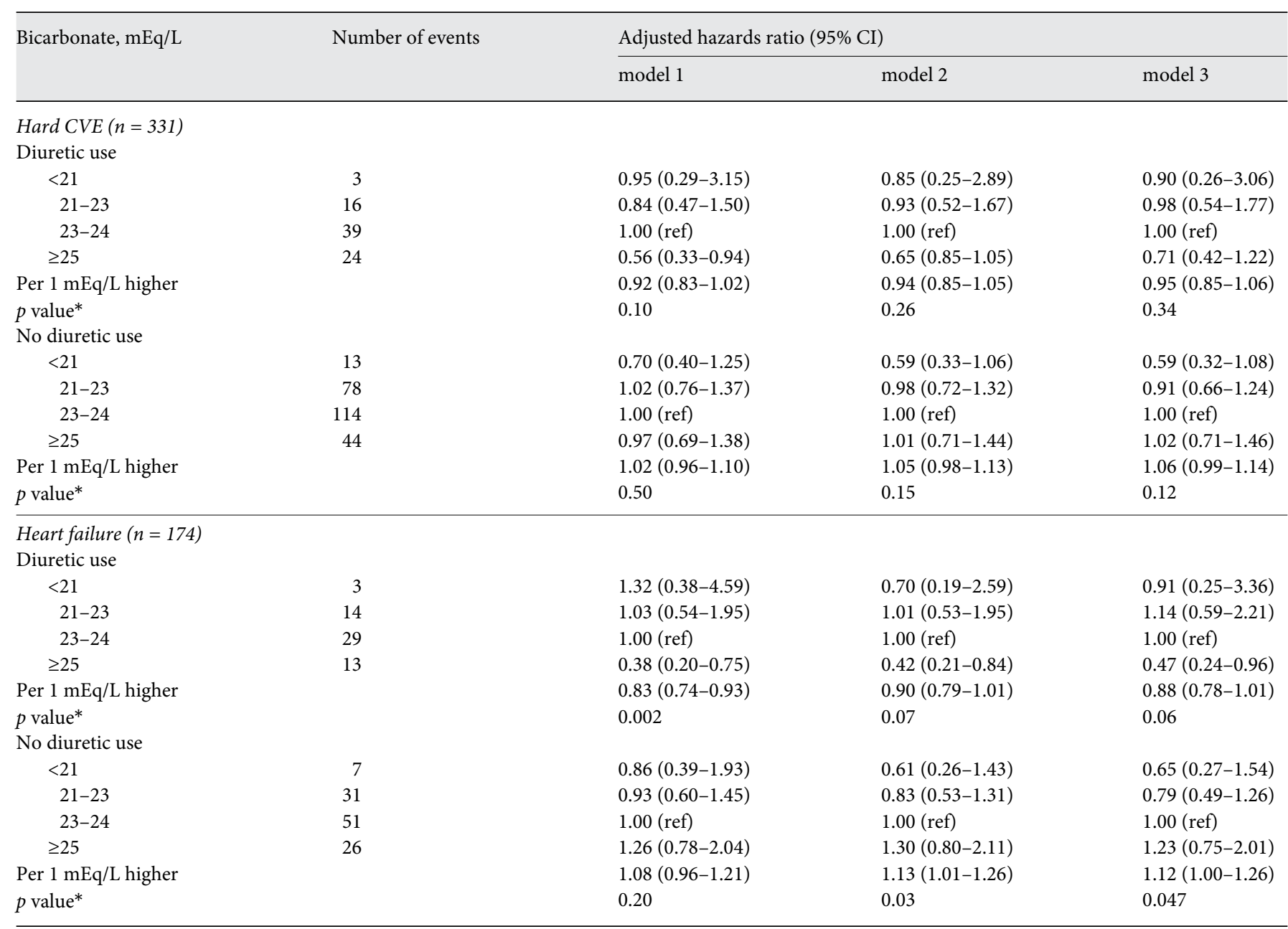

Model 1: adjusted for age, race/ethnicity, gender, education, and MESA site.

Model 2: adjusted for covariates in model 1 plus diabetes, self-reported emphysema, body mass index, systolic blood pressure, smoking, high-density lipoprotein cholesterol, low-density lipoprotein cholesterol, triglycerides, eGFR, C-reactive protein, urine albumin to creatinine ratio, use of antihypertensive medications, use of lipid-lowering medications.

Model 3: adjusted for covariates in model 2 plus dietary protein density. Interaction between diuretic use and bicarbonate examining incident heart failure ( $p$-interaction $=0.03$ in model 2 when bicarbonate was examined categorically and 0.003 in model 2 when bicarbonate was examined continuously).

* $p$ value is for continuous comparison.

were not significant in the categorical model. Results were unchanged after further adjustment for dietary protein density (model 3).

\section{Sensitivity Analysis}

Since lung function may affect serum bicarbonate, we performed an additional analysis using a more accurate measurement of emphysema (percent emphysema by CT) in the fully adjusted model. We included 3,622 participants in whom percent emphysema was available. Higher serum bicarbonate levels were still significantly associated with increased LVM and higher aortic PP. The direction of the association between bicarbonate and incident heart failure remained the same in participants not on diuretics, but was no longer statistically significant (For each $1 \mathrm{mEq} / \mathrm{L}$ increase in bicarbonate: hazards ratio 1.12 , 95\% CI $0.95-1.32$ ). This analysis had only 86 incident heart failure events compared with 174 in the primary analysis.

To determine whether bicarbonate concentrations were associated with a specific type of heart failure, we performed a sensitivity analysis examining the associa- 
tion of bicarbonate with preserved ejection fraction (EF) heart failure and reduced EF heart failure. Results are shown in online supplemental Table 1 (see www.karger. com/doi/10.1159/000454783). Bicarbonate concentration was not significantly associated with either subtype of heart failure in the fully adjusted analysis, but the direction of the association remained the same for both subtypes. The lack of a significant association may be due to the low number of events ( $n=69$ preserved EF events and $n=80$ reduced EF events).

\section{Discussion}

Contrary to our hypothesis, a higher serum bicarbonate concentration was associated with higher LVM, higher aortic PP and a higher risk of heart failure among nonusers of diuretics in a community-based, multiethnic cohort that was initially free of clinical CVD. We did not observe any association between bicarbonate concentration and incident atherosclerotic CVD events.

To our knowledge, this is the first study examining the relationship between serum bicarbonate and CVE in participants with generally normal kidney function. Our results are similar to 2 previous studies performed in the setting of CKD $[12,19]$. In a study of over 3,900 patients with CKD stages 2-4 from the Chronic Renal Insufficiency Cohort, each $1 \mathrm{mEq} / \mathrm{L}$ increase in bicarbonate concentration over $24 \mathrm{mEq} / \mathrm{L}$ was associated with a $14 \%$ increased risk of heart failure [12]. Additionally, the serum bicarbonate concentration was not associated with atherosclerotic CVD events. Similarly, an analysis from the RENAAL trial of over 2,600 participants with type 2 diabetes and CKD found that a bicarbonate level $\geq 27 \mathrm{mEq} / \mathrm{L}$ was associated with a higher risk of developing heart failure but not with a higher risk of atherosclerotic CVD events [19]. Together, these data suggest that higher serum bicarbonate may be a risk factor for heart failure, but not atherosclerotic CVD, among people with and without CKD.

It is important to note that the significant associations between serum bicarbonate concentrations and outcomes were observed only after detailed adjustment. Thus the association could be due to confounding. For example, it is possible that higher bicarbonate concentrations were actually a subclinical sign of heart failure rather than a causative factor. However, the high risk of heart failure observed in participants not on diuretics at higher serum bicarbonate concentrations could also be due, in part, to the effects of alkalosis on arterial stiffness and LV compliance, if we make the assumption that those with higher bicarbonate have higher $\mathrm{pH}$. We found that higher bicarbonate concentrations were associated with greater aortic PP (a measure of arterial stiffness) and LVM. It is hypothesized that metabolic alkalosis may promote vascular calcification and stiffness. An alkaline $\mathrm{pH}$ increases vascular calcification in cultured cells and uremic rats $[28,29]$ whereas acidosis inhibits calcification [30]. Experimental studies have demonstrated that acidosis prevents the production of hydroxyapatite and its soft tissue deposition by modulating the activity of several regulatory enzymes such as matrix gla-protein and alkaline phosphatase [31-34]. Vascular calcification induces arterial stiffness $[34,35]$ and arterial dysfunction is a key factor responsible for the development of LV hypertrophy [36]. Additionally, alkalosis activates specific mitogenactivated protein kinase signaling pathways that are involved in the regulation of cardiomyocyte cell survival and death [37].

Interestingly, we found that in participants on diuretics, a serum bicarbonate level $\geq 25 \mathrm{mEq} / \mathrm{L}$ was associated with a decreased risk of heart failure. While diuretics are a major cause of metabolic alkalosis [38], it is possible that elevated bicarbonate levels from diuretics are not harmful. Diuretics are the most effective class of drugs in preventing incident heart failure [39]. The unique preload reducing effect of diuretics may be the reason they are superior to other drugs at preventing heart failure. Hence, elevated bicarbonate levels that result from diuretics may be different than elevated levels from a different cause. Diuretics induce metabolic alkalosis due to a "contraction alkalosis" and by increasing net acid excretion in the distal tubule resulting in increased bicarbonate production [38]. Previous studies examining the association between bicarbonate concentration and heart failure were unable to stratify by diuretic use due to more than $60 \%$ of patients being on diuretics [12]. Future studies should examine whether elevated bicarbonate levels from diuretics are different than elevated bicarbonate levels from other etiologies.

While we did not find that lower serum bicarbonate concentrations are a risk factor for CVD or heart failure, lower serum bicarbonate concentrations may still be a risk factor for other outcomes. Lower serum bicarbonate has been shown to be a significant risk factor for progression of kidney disease in patients with established CKD and relatively preserved kidney function $[9-16,19]$. For example, in the MESA cohort, each 1-SD lower baseline bicarbonate concentration was associated with $12 \%$ higher odds of rapid kidney function decline [16]. Addition- 
ally, studies have shown that increasing serum bicarbonate through oral bicarbonate supplementation slows kidney function decline $[10,40,41]$. It is hypothesized that metabolic acidosis results in increases in aldosterone, endothelin, and angiotensin II levels leading to increased interstitial fibrosis and decline in GFR [10, 42]. Elevated levels of these hormones are also associated with CVD [43], but our results suggest that lower bicarbonate is not a risk factor for CVD and higher bicarbonate may promote heart failure. These conflicting data demonstrate the need for randomized clinical trials examining the effect of alkali therapy on vascular calcification, CVD, and kidney disease.

Our study has limitations worth mentioning. First, this is an observational study and thus we cannot prove causation. Second, we used a single measurement of baseline serum bicarbonate concentration. Third, we did not have data regarding the use of alkali therapy in the study participants. Additionally, our observed associations may be confounded by factors that were not measured in this study. For example, lung function may affect serum bicarbonate levels. However, we were able to adjust for selfreported emphysema as well as percent emphysema by CT scan in a subset of patients and the relationships of bicarbonate persisted with LVM, aortic stiffness and heart failure. Finally, we did not have a complete description of the acid-base status of the patients $\left(\mathrm{pH}, \mathrm{PCO}_{2}\right.$, and bicarbonate level), and thus, we do not know if the patients with higher serum bicarbonate levels were alkalemic. Studies have shown that patients can have alkalemia or acidemia with various serum bicarbonate concentrations [18] and future studies should obtain a complete acid-base profile.

Our study also has several strengths including the large number of participants, long-term follow-up, adjudicat- ed CVE, and complementary measurements of subclinical CVD. Also, we examined a population free of clinical CVD at baseline, reducing risks of reverse causation, and confounding. Finally, we adjusted for established cardiovascular risk factors as well as lung function.

In conclusion, we found that higher serum bicarbonate concentrations were associated with increased risks of subclinical CVD and clinical heart failure outcomes in a large, multiethnic, community-based cohort study. Our findings suggest that higher bicarbonate concentrations may be a risk factor for arterial stiffness, LVM, and heart failure. Further studies are needed to determine relationships of bicarbonate concentrations and bicarbonate interventions with cardiovascular outcomes.

\section{Acknowledgments}

This work was supported by the National Institute of Diabetes and Digestive and Kidney Disease grants K23 DK087859, 1R01DK081473, R01DK088762, and R01DK099199, R01DK098234, National Heart, Lung, and Blood Institute grant R01HL096875, and an Established Investigator Award from the American Heart Association (14EIA18560026). This research was supported by contracts HHSN268201500003I, N01-HC-95159, N01-HC-95160, N01-HC-95161, N01-HC-95162, N01-HC-95163, N01-HC-95164, N01-HC-95165, N01-HC-95166, N01-HC-95167, N01-HC-95168, and N01-HC-95169 from the National Heart, Lung, and Blood Institute and by grants UL1-TR-000040 and UL1-TR-001079 from NCRR. The authors thank the other investigators, the staff, and the participants of the MESA study for their valuable contributions. A full list of participating MESA investigators and institutions can be found at http://www.mesa-nhlbi.org.

\section{Disclosure Statement}

Authors do not have any disclosures.

\section{References}

1 Writing Group Members, Mozaffarian D, Benjamin EJ, Go AS, Arnett DK, Blaha MJ, Cushman M, Das SR, de Ferranti S, Despres JP, Fullerton HJ, Howard VJ, Huffman MD, Isasi CR, Jimenez MC, Judd SE, Kissela BM, Lichtman JH, Lisabeth LD, Liu S, Mackey RH, Magid DJ, McGuire DK, Mohler ER 3rd, Moy CS, Muntner P, Mussolino ME, Nasir K, Neumar RW, Nichol G, Palaniappan L, Pandey DK, Reeves MJ, Rodriguez CJ, Rosamond W, Sorlie PD, Stein J, Towfighi A, Turan TN, Virani SS, Woo D, Yeh RW, Turner MB: Heart disease and stroke statistics-2016 update: a report from the American Heart Association. Circulation 2016;133:e38-e360.
2 Kopple JD, Kalantar-Zadeh K, Mehrotra R: Risks of chronic metabolic acidosis in patients with chronic kidney disease. Kidney Int Suppl 2005;95:S21-S27.

3 Wesson DE, Simoni J, Broglio K, Sheather S: Acid retention accompanies reduced GFR in humans and increases plasma levels of endothelin and aldosterone. Am J Physiol Renal Physiol 2011;300:F830-F837.

4 MandelEI, Forman JP, Curhan GC, Taylor EN: Plasma bicarbonate and odds of incident hypertension. Am J Hypertens 2013;26:14051412 .

5 Forman JP, Rifas-Shiman SL, Taylor EN, Lane K, Gillman MW: Association between the serum anion gap and blood pressure among patients at Harvard Vanguard Medical Associates. J Hum Hypertens 2008;22: 122-125.

6 Taylor EN, Forman JP, Farwell WR: Serum anion gap and blood pressure in the national health and nutrition examination survey. Hypertension 2007;50:320-324.

7 Farwell WR, Taylor EN: Serum bicarbonate, anion gap and insulin resistance in the National Health and Nutrition Examination Survey. Diabet Med 2008;25:798-804.

8 Mandel EI, Curhan GC, Hu FB, Taylor EN Plasma bicarbonate and risk of type 2 diabetes mellitus. CMAJ 2012;184:E719-E725. 
9 Shah SN, Abramowitz M, Hostetter TH, Melamed ML: Serum bicarbonate levels and the progression of kidney disease: a cohort study. Am J Kidney Dis 2009;54:270-277.

10 Phisitkul S, Khanna A, Simoni J, Broglio K, Sheather S, Rajab MH, Wesson DE: Amelioration of metabolic acidosis in patients with low GFR reduced kidney endothelin production and kidney injury, and better preserved GFR. Kidney Int 2010;77:617-623.

11 Goldenstein L, Driver TH, Fried LF, Rifkin DE, Patel KV, Yenchek RH, Harris TB, Kritchevsky SB, Newman AB, Sarnak MJ, Shlipak MG, Ix JH; Health ABC Study Investigators: Serum bicarbonate concentrations and kidney disease progression in community-living elders: the health, aging, and body composition (Health ABC) Study. Am J Kidney Dis 2014;64:542-549.

12 Dobre M, Yang W, Chen J, Drawz P, Hamm LL, Horwitz E, Hostetter T, Jaar B, Lora CM, Nessel L, Ojo A, Scialla J, Steigerwalt S, Teal V, Wolf M, Rahman M; CRIC Investigators: Association of serum bicarbonate with risk of renal and cardiovascular outcomes in CKD: a report from the Chronic Renal Insufficiency Cohort (CRIC) study. Am J Kidney Dis 2013; 62:670-678.

13 Kanda E, Ai M, Yoshida M, Kuriyama R, Shiigai T: High serum bicarbonate level within the normal range prevents the progression of chronic kidney disease in elderly chronic kidney disease patients. BMC Nephrol 2013;14:4.

14 Raphael KL, Wei G, Baird BC, Greene T, Beddhu S: Higher serum bicarbonate levels within the normal range are associated with better survival and renal outcomes in African Americans. Kidney Int 2011;79:356-362.

15 Kovesdy CP, Anderson JE, Kalantar-Zadeh K: Association of serum bicarbonate levels with mortality in patients with non-dialysis-dependent CKD. Nephrol Dial Transplant 2009; 24:1232-1237.

16 Driver TH, Shlipak MG, Katz R, Goldenstein L, Sarnak MJ, Hoofnagle AN, Siscovick DS, Kestenbaum B, de Boer IH, Ix JH: Low serum bicarbonate and kidney function decline: the Multi-Ethnic Study of Atherosclerosis (MESA). Am J Kidney Dis 2014;64:534-541.

17 Navaneethan SD, Schold JD, Arrigain S, Jolly SE, Wehbe E, Raina R, Simon JF, Srinivas TR, Jain A, Schreiber MJ Jr, Nally JV Jr: Serum bicarbonate and mortality in stage 3 and stage 4 chronic kidney disease. Clin J Am Soc Nephrol 2011;6:2395-2402.

18 Raphael KL, Murphy RA, Shlipak MG, Satterfield S, Huston HK, Sebastian A, Sellmeyer DE, Patel KV, Newman AB, Sarnak MJ, Ix JH, Fried LF; Health ABC Study: Bicarbonate concentration, acid-base status, and mortality in the health, aging, and body composition study. Clin J Am Soc Nephrol 2016;11:308-316.

19 Schutte E, Lambers Heerspink HJ, Lutgers HL, Bakker SJ, Vart P, Wolffenbuttel BH,
Umanath K, Lewis JB, de Zeeuw D, Gansevoort RT: Serum bicarbonate and kidney disease progression and cardiovascular outcome in patients with diabetic nephropathy: a post hoc analysis of the RENAAL (Reduction of End Points in Non-Insulin-Dependent Diabetes with the Angiotensin II Antagonist Losartan) Study and IDNT (Irbesartan Diabetic Nephropathy Trial). Am J Kidney Dis 2015;66:450-458.

20 Bild DE, Bluemke DA, Burke GL, Detrano R, Diez Roux AV, Folsom AR, Greenland P, Jacob DR Jr, Kronmal R, Liu K, Nelson JC, O'Leary D, Saad MF, Shea S, Szklo M, Tracy RP: Multi-Ethnic Study of Atherosclerosis: objectives and design. Am J Epidemiol 2002; 156:871-881.

21 Bosworth C, Sachs MC, Duprez D, Hoofnagle AN, Ix JH, Jacobs DR Jr, Peralta CA, Siscovick DS, Kestenbaum B, de Boer IH: Parathyroid hormone and arterial dysfunction in the multi-ethnic study of atherosclerosis. Clin Endocrinol (Oxf) 2013;79:429-436.

22 Natori S, Lai S, Finn JP, Gomes AS, Hundley WG, Jerosch-Herold M, Pearson G, Sinha S, Arai A, Lima JA, Bluemke DA: Cardiovascular function in multi-ethnic study of atherosclerosis: normal values by age, sex, and ethnicity. AJR Am J Roentgenol 2006;186(6 suppl 2):S357-S365.

23 Martin SS, Blaha MJ, Muse ED, Qasim AN, Reilly MP, Blumenthal RS, Nasir K, Criqui MH, McClelland RL, Hughes-Austin JM, Allison MA: Leptin and incident cardiovascular disease: the Multi-ethnic Study of Atherosclerosis (MESA). Atherosclerosis 2015;239:67-72.

24 Dharod A, Soliman EZ, Dawood F, Chen H, Shea S, Nazarain S, Bertoni AG; MESA Investigators: Association of asymptomatic bradycardia with incident cardiovascular disease and mortality: the Multi-Ethnic Study of Atherosclerosis (MESA). JAMA Intern Med 2016;176:219-227.

25 Criqui MH, Denenberg JO, Ix JH, McClelland RL, Wassel CL, Rifkin DE, Carr JJ, Budoff MJ, Allison MA: Calcium density of coronary artery plaque and risk of incident cardiovascular events. JAMA 2014;311:271-278.

26 Nettleton JA, Polak JF, Tracy R, Burke GL, Jacobs DR Jr: Dietary patterns and incident cardiovascular disease in the Multi-Ethnic Study of Atherosclerosis. Am J Clin Nutr 2009;90: 647-654.

27 Remer T, Manz F: Potential renal acid load of foods and its influence on urine $\mathrm{pH}$. J Am Diet Assoc 1995;95:791-797.

28 Lomashvili K, Garg P, O’Neill WC: Chemical and hormonal determinants of vascular calcification in vitro. Kidney Int 2006;69:14641470.

29 de Solis AJ, Gonzales-Pacheco FR, Deudero JJ, Neria F, Albalate M, Petkov V, Susanibar L, Fernandez-Sanchez R, Calabia O, Ortiz A, Caramelo C: Alkalinization potentiates vas- cular calcium deposition in an uremic milieu. J Nephrol 2009;22:647-653.

30 Mendoza FJ, Lopez I, Montes de Oca A, Perez J, Rodriguez M, Aguilera-Tejero E: Metabolic acidosis inhibits soft tissue calcification in uremic rats. Kidney Int 2008;73:407-414.

31 Yonova D: Vascular calcification and metabolic acidosis in end stage renal disease. Hippokratia 2009;13:139-140.

32 Frick KK, Jiang L, Bushinsky DA: Acute metabolic acidosis inhibits the induction of osteoblastic egr- 1 and type 1 collagen. Am J Physiol 1997;272(5 pt 1):C1450-C1456.

33 Frick KK, Bushinksy DA: Chronic metabolic acidosis reversibly inhibits extracellular matrix gene expression in mouse osteoblasts. Am J Physiol 1998;275(5 pt 2):F840-F847.

34 Haydar AA, Covic A, Colhoun H, Rubens M, Goldsmith DJ: Coronary artery calcification and aortic pulse wave velocity in chronic kidney disease patients. Kidney Int 2004;65: 1790-1794.

35 Guerin AP, London GM, Marchais SJ, Metivier F: Arterial stiffening and vascular calcifications in end-stage renal disease. Nephrol Dial Transplant 2000;15:1014-1021.

36 Glassock RJ, Pecoits-Filho R, Barberato SH Left ventricular mass in chronic kidney disease and ESRD. Clin J Am Soc Nephrol 2009;(4 suppl 1):S79-S91.

37 Stathopoulou K, Beis I, Gaitanaki C: MAPK signaling pathways are needed for survival of H9c2 cardiac myoblasts under extracellular alkalosis. Am J Physiol Heart Circ Physiol 2008;295:H1319-H1329.

38 Palmer BF: Metabolic complications associated with use of diuretics. Semin Nephrol 2011;31:542-552.

39 Sciarretta S, Palano F, Tocci G, Baldini R, Volpe M: Antihypertensive treatment and development of heart failure in hypertension: a Bayesian network meta-analysis of studies in patients with hypertension and high cardiovascular risk. Arch Intern Med 2011;171:384394

40 de Brito-Ashurst I, Varagunam M, Raftery MJ, Yaqoob MM: Bicarbonate supplementation slows progression of CKD and improves nutritional status. J Am Soc Nephrol 2009;20: 2075-2084

41 Mahajan A, Simoni J, Sheather SJ, Broglio KR, Rajab MH, Wesson DE: Daily oral sodium bicarbonate preserves glomerular filtration rate by slowing its decline in early hypertensive nephropathy. Kidney Int 2010;78: 303-309.

42 Kraut JA, Madias NE: Metabolic acidosis of CKD: an update. Am J Kidney Dis 2016;67: 307-317.

43 Ruiz-Ortega M, Lorenzo O, Ruperez M, Esteban V, Suzuki Y, Mezzano S, Plaza JJ, Egido J: Role of the renin-angiotensin system in vascular diseases: expanding the field. Hypertension 2001;38:1382-1387. 